\title{
Metformin-Induced Mitochondrial Complex I Inhibition: Facts, Uncertainties, and Consequences
}

\author{
Eric Fontaine* \\ INSERM, LBFA, Université Grenoble Alpes, Grenoble, France
}

Metformin is the most widely prescribed drug to treat patients with type II diabetes, for whom retrospective studies suggest that metformin may have anticancer properties. However, in experiments performed with isolated cells, authors have reported both pro- and anti-apoptotic effects of metformin. The exact molecular mechanism of action of metformin remains partly unknown despite its use for over 60 years and more than 17,000 articles in PubMed. Among the various widely recognized or recently proposed targets, it has been reported consistently that metformin is capable of inhibiting mitochondrial respiratory chain Complex I. Since most of the effects of metformin have been replicated by other inhibitors of Complex I, it has been suggested that the mechanism of action of metformin involved the inhibition of Complex I. However,

OPEN ACCESS

Edited by:

Frederic Bost

Centre National de la Recherche

Scientifique (CNRS), France

Reviewed by:

Gayathri K. Natarajan,

Medical College of Wisconsin,

United States

Adam Mickiewicz,

University in Poznan, Poland

${ }^{*}$ Correspondence: Eric Fontaine

eric.fontaine@univ-grenoble-alpes.fr

Specialty section:

This article was submitted to Cellular Endocrinology,

a section of the journal

Frontiers in Endocrinology

Received: 27 April 2018 Accepted: 27 November 2018 Published: 17 December 2018

Citation: Fontaine E (2018) Metformin-Induced Mitochondrial Complex I Inhibition:

Facts, Uncertainties, and Consequences.

Front. Endocrinol. 9:753. doi: 10.3389/fendo.2018.00753 compared to conventional Complex I inhibitors, the metformin-induced inhibition of Complex I has unique characteristics. Among these, the most original one is that the concentrations of metformin required to inhibit Complex I are lower in intact cells than in isolated mitochondria. Experiments with isolated mitochondria or Complex I were generally performed using millimolar concentrations of metformin, while plasma levels remain in the micromolar range in both human and animal studies, highlighting that metformin concentration is an important issue. In order to explain the effects in animals based on observations in cells and mitochondria, some authors proposed a direct effect of the drug on Complex I involving an accumulation of metformin inside the mitochondria while others proposed an indirect effect (the drug no longer having to diffuse into the mitochondria). This brief review attempts to: gather arguments for and against each hypothesis concerning the mechanism by which metformin inhibits Complex I and to highlight remaining questions about the toxicity mechanism of metformin for certain cancer cells.

\section{Keywords: metformin, mitochondria, Complex I, pharmacokinetic, cell death, cancer, permeability transition}

\section{INTRODUCTION}

Metformin is a drug with pleiotropic effects. It takes part in glucose homeostasis, mainly by inhibiting liver glucose production (1). It also modifies the production of reactive oxygen species and affects cell death processes $(2,3)$. Most of these effects have been traced to the inhibition of mitochondrial respiratory chain Complex I for two main reasons: First, over the past 20 years, different laboratories have reproducibly observed that metformin inhibits mitochondrial respiratory chain Complex I (4-20). Second, these pleiotropic effects have been reproduced by well identified Complex I inhibitors [gluconeogenesis $(21,22)$, cell death $(18,23-28)$ ]. 
However, the mechanism by which metformin affects the activity of Complex I remains debated. In order to clarify whether the different conclusions found in the literature may be due to methodological differences, this review compares results obtained in vivo or with intact cells, to results obtained with isolated mitochondria or isolated Complex I. In this last case, authors tend to assume that metformin accumulates in mitochondria, here we will discuss evidence supporting or not this assumption. Finally, since pro- and anti-apoptotic effects of metformin are observed in intact cells, we will examine the role of metformin concentrations as a potential cause of these conflicting observations.

\section{METFORMIN PHARMACOKINETICS}

Metformin is a hydrophilic compound charged positively at physiological $\mathrm{pH}$. Its hydrophilicity limits its permeability through lipid membranes. Metformin enters and leaves cells by the presence of several transporters including Organic Cation Transporters (OCTs) and multidrug and toxin extrusion (MATE) transporters (29). This leads to a steady-state concentration of metformin inside cells, depending on both the amount and activity of such transporters as well as metformin plasma concentration.

The pharmacological inhibition or the genetic ablation of OCTs reduce the distribution of metformin to the liver, small intestine and kidney (30-32) while the overexpression of OCT1 in HEK293 and CHO cells increases metformin uptake $(30,33)$. The pharmacological inhibition or the genetic ablation of MATE1 cause hepatic and kidney accumulation of metformin $(32,34)$. In humans, the genomic variations of metformin transporters can affect its pharmacokinetics (concentration, clearance, volume of distribution) $(35,36)$ suggesting that such genomic variations affect metformin concentration in tissues.

Whether the activities of the metformin transporters (i.e., the metformin concentration in tissues) affect the metabolic effects of metformin is not systematically reported in the literature. On the one hand, metformin failed to reduce fasting plasma glucose concentration in OCT1-knockout mice submitted to a highfat diet for 8 weeks and failed to suppress glucagon-stimulated glucose production in $\mathrm{OCT}^{-} /^{-}$hepatocytes $(30)$. On the other hand, the effect of metformin on glucose tolerance tests was similar in animal controls and OCT1/2-knockout animals (31). A broad variation in clinical efficacy of metformin has long been recognized as well as a reduced function polymorphism of OCT1 in humans. However, if some authors reported a decreased effect of metformin in type- 2 diabetes patients carrying reduced function polymorphism of OCT1 $(30,36)$, others did not observe such a correlation $(37,38)$.

To the best of my knowledge, no study correlating metformin concentration in tissue (or cells) and metformin-induced Complex I inhibition was ever published.

Drugs that are extensively sequestered in organelles have a very large apparent volume of distribution and a prolonged halflife in vivo (39). Metformin is not metabolized and is secreted by the kidneys with a half-life of 1.74-7.3 h in humans depending on the studies $(35,40-42)$. With a volume of distribution of $1.12 \pm$ $0.08 \mathrm{~L} / \mathrm{kg}$ in healthy volunteers (40), metformin is not supposed to accumulate dramatically in tissues. The amount of metformin in the liver ranges from 2 to 5 times that of plasma -depending on the studies $(32,35,42,43)$ - and increases up to 10 times that of plasma in small intestinal walls (32).

Thus, the pharmacokinetic studies indicate that metformin enters but does not accumulate in large amounts in cells. Whether its metabolic activity depends on its diffusion inside the cells is supported by several but not all studies.

Once in the cell, as metformin inhibits Complex I it is tempting to speculate that metformin penetrates the mitochondria. The composition of the mitochondrial matrix (the space delimited by the inner mitochondrial membrane) is different from that of the cytosol. In order to maintain such a different metabolite composition, the inner membrane is impermeable to almost all hydrophilic molecules which enter or leave the mitochondria through specific transporters. Among the numerous recognized mitochondrial carriers, no specific carrier for metformin has been identified yet.

Despite this, many authors have hypothesized that metformin accumulates in mitochondria $(5,13,15,44)$. This scenario may reconcile the observation that millimolar concentrations of metformin are necessary to inhibit Complex I in isolated mitochondria (see below) while, when used at the therapeutic dose, the plasma metformin concentration remains in the micromolar range in both humans and animals $(31,36,42)$.

From a theoretical point of view, this hypothesis is plausible. Indeed, because the mitochondrial respiratory chain transfers protons from the matrix to the intermembrane space, mitochondria build up and maintain an electrical mitochondrial membrane potential that drives the accumulation of positively charged molecules into mitochondria, provided the molecule crosses the membrane. In these conditions, Nernst equation indicates that for a physiological mitochondrial membrane potential of $-180 \mathrm{mV}$ the thermodynamic equilibrium is reached after a 1,000-fold accumulation of a positively charged molecule if the molecule has one charge. Since metformin is a positively charged molecule and assuming the presence of a still unknown carrier for metformin in the inner membrane, its mitochondrial concentration could reach the millimolar range despite a cytosolic concentration within the micromolar range (see Figure 1). In addition, assuming a plasma membrane potential of $-36 \mathrm{mV}$ and the absence of kinetic constraints on metformin transporters (OCT and MATE), the cytosolic concentration of metformin would be 4 times that of plasma.

However, the hypothesis that metformin accumulates in mitochondria contradicts several observations.

First of all, the accumulation of numerous positive charges in the matrix compensated by proton extrusion by the respiratory chain, should lead to a collapse of mitochondrial membrane potential associated with an increase in delta $\mathrm{pH}$. However, note that metformin did not depolarize isolated mitochondria (8).

Secondly, assuming that the total mitochondrial volume represents approximately $20 \%$ of hepatocytes, a 1,000-fold accumulation of metformin inside mitochondria would represent an approximately 200 -fold accumulation of metformin in liver 
Hypothesis of a direct action on Complex I

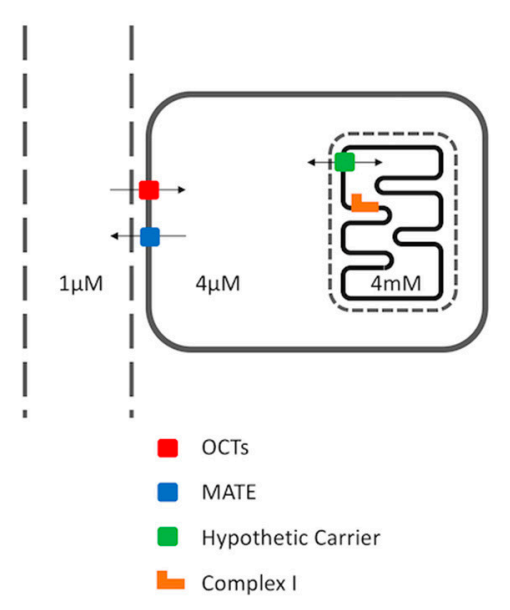

Hypothesis of an indirect action on Complex I

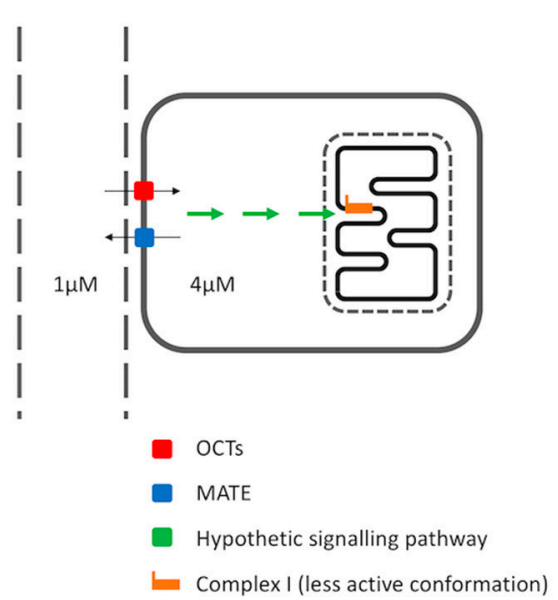

FIGURE 1 | Mechanism of action of metformin on complex I: the direct hypothesis and the indirect hypothesis. Metformin enters cells via Organic Cation Transporters (OCTs) and leaves cells via multidrug and toxin extrusion (MATE) transporters. Assuming a plasma membrane potential of $35 \mathrm{mV}$ and a passive mechanism of OCTs and MATE, the cytosolic metformin concentration is expected to be 4 times that of the plasma concentration. In the direct hypothesis (left), metformin is supposed to enter mitochondria via a hypothetical carrier reaching a matrix concentration 1,000 times that of the cytosol for mitochondrial membrane potential of $180 \mathrm{mV}$. In the indirect hypothesis (right), metformin does not enter mitochondria but stimulates a hypothetical signaling pathway that eventually modifies Complex I conformation, making it less active.

(without accounting for accumulation in the cytosol). Such an accumulation is 2 orders of magnitude higher than that measured by several groups $(32,35,42,43)$.

Thirdly, a large mitochondrial accumulation is not compatible with the low volume of distribution of metformin and its short half-live (see above).

Fourthly, using radioactive $\left[{ }^{14} \mathrm{C}\right]$ metformin, the radioactivity was not found to accumulate in liver mitochondria of rats treated orally with metformin (45) and no radioactivity was measured inside mitochondria when Xenopus laevis oocytes were exposed to concentrations of metformin that led to Complex I inhibition (6). Importantly, Complex I remained inhibited after mitochondrial isolation. Although this result does not definitively exclude a possible accumulation of metformin in mitochondria as a cause of Complex I inhibition (accumulated metformin may diffuse during the isolation procedure), it rules out the hypothesis that the inhibition of Complex I by metformin requires metformin inside mitochondria.

In summary, unlike the less hydrophilic biguanides (46), the accumulation of metformin inside the mitochondria is not supported by direct measurements, is not consistent with the pharmacokinetic data, and would require a transporter that has not yet been discovered.

Derivatives combining a molecule of metformin at different alkyl chain lengths containing a triphenylphosphonium cation (a liposoluble cation known to accumulate in mitochondria according to membrane potential) have been synthesized (47, 48 ) in order to increase the anti-cancer effect of metformin (see below). These different compounds accumulate in cells (47), depolarize mitochondria (48) and inhibit Complex I with an $\mathrm{IC}_{50}$ in the micromolar range $(47,48)$, which according to Nernst equation is consistent with the accumulation of compounds in the mitochondrial matrix at a concentration in the millimolar range. If metformin accumulated spontaneously in the mitochondria, the addition of molecules targeting the mitochondria would be unnecessary, which is clearly not the case.

\section{CHARACTERISTICS OF COMPLEX I INHIBITION ACCORDING TO THE MODELS USED}

Although this may seem odd, it has been reported by several different laboratories that the concentrations required to inhibit Complex I are lower for intact cells than for isolated mitochondria $(4-6,13,19)$. Note however that the characteristics of Complex I inhibition reveals some differences depending on whether metformin acts on intact cells (animal models, infused organs, isolated cells) or directly on isolated mitochondria or isolated Complex I (see Table 1).

The incubation of isolated Complex I or submitochondrial particles in the presence of millimolar concentrations of metformin leads to an inhibition of Complex I that can be complete (13) with an $\mathrm{IC}_{50}$ ranging from 19 to $79 \mathrm{mM}$ depending on laboratories $(5,9,13)$. It should be noted that there is no membrane potential in these particular conditions of incubation, thus no possibility of metformin accumulation. In other words, the concentrations tested are the actual concentrations to which Complex I is exposed.

The incubation of isolated mitochondria in the presence of millimolar concentrations of metformin leads to a rather fast (within a few minutes) inhibition of Complex I with an “apparent" $\mathrm{IC}_{50}$ also in the millimolar range (5). 
TABLE 1 | Main differences in the characteristics of Complex I inhibition according to the model used.

\begin{tabular}{|c|c|c|c|c|}
\hline & \multicolumn{2}{|c|}{ Live animal, perfused organ, intact cells } & \multicolumn{2}{|c|}{ Isolated mitochondria, isolated Complex I } \\
\hline & & References & & References \\
\hline Speed of inhibition & Time dependent & (4) (5) & Immediate (minutes) & $(14)(13)$ \\
\hline Type of inhibition & Partial & (4) (5) (18) & Total & $(13)$ \\
\hline Affinity & $\begin{array}{l}\text { Apparent } \mathrm{IC}_{50} \text { approximately } 1 \mathrm{mM} \text { after } \\
30 \text { min in isolated rat hepatocytes } \\
\text { Apparent } \mathrm{IC}_{50} 250 \mu \mathrm{M} \text { and } 330 \mu \mathrm{M} \text { after } \\
6 \mathrm{~h} \text { incubation in } 143 \mathrm{~B} \text { and HepG } 2 \text { cells } \\
\text { respectively }\end{array}$ & $\begin{array}{l}(4) \\
(13)\end{array}$ & $\begin{array}{l}\text { Apparent } I_{50} 15 \mathrm{mM} \text { in isolated } \\
\text { mitochondria } \\
I_{50} 79 \mathrm{mM} \text { in sub-mitochondrial particles } \\
I_{5} C_{50} 66 \mathrm{mM} \text { in immunocaptured Complex I } \\
I_{50} 19 \mathrm{mM} \text { in isolated Complex I }\end{array}$ & $\begin{array}{l}\text { (5) } \\
(5) \\
(9) \\
(13)\end{array}$ \\
\hline Inhibition in State-3? & Yes & $\begin{array}{l}(5)(11)(12) \\
(14)(15)\end{array}$ & Yes & $\begin{array}{l}(5)(8)(9)(10) \\
(12)(14)(15)\end{array}$ \\
\hline Inhibition in State-4? & Yes & (4) (7) (14) & No & (4) (8) (10) (15) \\
\hline $\begin{array}{l}\text { Inhibition after } \\
\text { uncoupling }\end{array}$ & Yes & $\begin{array}{l}(4)(7)(11)(14) \\
(19)\end{array}$ & No & (4) (15) (19) \\
\hline $\begin{array}{l}\text { Inhibition after the } \\
\text { removal of metformin }\end{array}$ & Yes & (4) (14) (20) & No for metformin concentration $\leq 2 \mathrm{mM}$ & $(13)$ \\
\hline $\mathrm{NADH} / \mathrm{NAD}^{+}$ & Increases & (4) (5) (18) & Decreases & (19) \\
\hline
\end{tabular}

This observation is not easily reconcilable with the proposal that metformin accumulates in mitochondria. Indeed, assuming that metformin did accumulate in mitochondria, Complex I inhibition would have been observed at micromolar concentrations of metformin (corresponding to millimolar concentrations inside mitochondria), which has not been reported. One could argue that at millimolar concentrations of metformin, the inhibition of Complex I would depolarize mitochondria, preventing metformin accumulation. However, it has been shown that millimolar concentrations of metformin did not depolarize isolated mitochondria (8).

In these particular conditions of incubation (isolated mitochondria exposed to millimolar concentrations of metformin), it has to be noted that the inhibition of Complex I is observed almost exclusively during ATP synthesis (also called State 3) and disappears when mitochondria are depolarized (uncoupled State) or at rest (also called State 4) $(4,8,15)$. Such behavior is not observed with rotenone (the reference inhibitor of Complex I) but is typical of biguanide-induced inhibition of Complex I in isolated mitochondria (49).

It has been proposed that the reason why the inhibition is not observed after uncoupling might be due to the fact that the driving force for metformin accumulation within mitochondria disappears in these particular conditions. Although, as stated above, the accumulation of metformin in mitochondria is not supported by any evidence, this hypothesis does not explain the lack of inhibition in State 4, a situation in which the driving force (the membrane potential) is higher than in State 3.

Curiously, it has been reported that the inhibition of oxygen consumption in isolated mitochondria is accompanied by NADH oxidation (19). This observation is not expected in case of a simple Complex I inhibition, suggesting an uncoupling effect of metformin in this particular condition.

The incubation of intact cells in the presence of metformin leads to a slower inhibition of Complex I depending on metformin concentration (hours are required for micromolar concentrations of metformin) $(5,50)$. Contrary to what is observed in isolated Complex I, the inhibition is not total and plateaus at approximately $40 \%$ of the $V \max (4)$. Consistent with a pure effect on Complex I, the inhibition leads to an increase in the $\mathrm{NADH} / \mathrm{NAD}^{+}$ratio (as assessed by the Lactate/pyruvate and 3 -hydroxybutyrate/ acetoacetate ratios) $(4,5)$. Importantly, once cells are permeabilized (i.e., once mitochondria can be studied as if they were isolated) the inhibition is observed in State 3, but also in State 4 and after uncoupling $(4,11,19)$. Finally, Complex I remains inhibited in mitochondria isolated from either rat exposed to metformin or liver perfused with metformin, even after uncoupling $(4,14)$ or when $\mathrm{NADH}$ :quinone oxidoreductase activity (i.e., Complex I activity) is studied directly using broken mitochondria (4). Note that the isolation procedure removes most of (if not all) the free metformin, while uncoupling (either chemical or after inner membrane rupture) would release the putative accumulated metformin. Although these results do not exclude a possible binding of metformin in mitochondrial membrane, they rule out the hypothesis that the inhibition of Complex I by metformin could depend on membrane potential.

\section{OTHER MITOCHONDRIAL EFFECTS OF METFORMIN}

In intact cells the inhibition of oxygen consumption is strictly located on Complex I. This conclusion comes from the observation that metformin has no effect on oxygen consumption when electrons feed the respiratory chain downstream Complex I (using succinate for example) regardless of the respiratory State (3, 4 and uncoupled) (4).

On the contrary, using isolated mitochondria and millimolar concentrations of metformin, some authors reported inhibitory effects on complexes III and IV (16). High concentrations of metformin have been reported to inhibit ATP hydrolysis but not 
ATP synthesis (13), suggesting an unconventional effect on the ATP synthase.

Some evidence suggests that Complex I can interact with ATP synthase (51). So we may infer that in this particular condition of incubation (isolated mitochondria exposed to millimolar concentrations of metformin), ATP synthesis possibly sensitizes Complex I to metformin. Although speculative, this personal suggestion could account for the observation that millimolar concentrations of metformin inhibit Complex I almost exclusively in State 3.

In summary, on intact cells metformin acts slowly but the effect is visible at micromolar concentrations. The inhibition affects only Complex I in all the respiratory states and does not depend on mitochondrial membrane potential. On isolated mitochondria (or isolated Complex I), metformin acts rapidly but the effect requires millimolar concentrations. The inhibition does not only affect Complex I and Complex I inhibition is not observed in all the respiratory states.

\section{WHERE DOES METFORMIN ACT ON COMPLEX I?}

The respiratory chain is a sequence of redox reactions which couple an electron flux with a vectorial transfer of protons. Mammalian respiratory chain complex $\mathrm{I}$ is a large protein complex with at least 45 subunits. It includes a hydrophobic part embedded in the inner membrane involved in proton transfer and a hydrophilic part protruding into the matrix in which electrons pass from NADH to ubiquinone via a succession of redox reactions. Complex I inhibitors rotenone and piericidin bind at, or close to, the ubiquinone binding site, inhibiting both electron flux and proton extrusion. Using artificial electron acceptors, a rotenone-insensitive NADH oxidation which is not coupled with proton pumping (i.e., a non-physiological pathway) can occur in Complex I.

Using isolated Complex I and millimolar concentrations of metformin, it has been shown that metformin does not inhibit $\mathrm{NADH}$ oxidation due to artificial electron acceptors, behaves as a non-competitive inhibitor of the physiological electron pathway and preferentially binds Complex I when the enzyme is in its "deactive" conformation (13). However, the exact localization where metformin acts in this condition of incubation remains unknown. Moreover, the exact mechanism leading to the inhibition of Complex I in intact cells using micromolar concentrations of metformin and where exactly it inhibits the electron flux in Complex I has not been reported.

\section{HYPOTHETICAL MECHANISMS OF ACTION}

To account for the fact that the concentration of metformin required to observe the inhibition of Complex I on whole cells is lower than the concentration required to observe the inhibition on mitochondria, two hypotheses have been proposed in the literature (see Figure 1).
The first one (in chronological order, but second in popularity) proposes that in vivo and in intact cells, metformin triggers a signaling pathway that in turn induces the inhibition of Complex I (4). Although such a signaling pathway is yet unknown, it has been reported that Complex I exists in two different functional conformations (active and inactive) (52), while reactive thiols of several Complex I subunits have been identified as targets for post-translational modifications (53, 54). However, whether metformin affects reactive thiols in Complex I has not been published yet.

The second hypothesis necessarily involves an accumulation of metformin in the mitochondria that would be driven by mitochondrial membrane potential. Although proposed by several authors, this hypothesis is not yet supported by any evidence (see above).

\section{EFFECTS OF METFORMIN-INDUCED COMPLEX I INHIBITION ON CELL DEATH PROCESSES}

Apparently contradictory effects are found in the literature regarding the effects of metformin on cell death. Some authors have put forward its protective effects against cell death (3) while others have reported its induction of cell death especially in cancer cells (2). Yet, all of them have concluded that the observed effects are due to the inhibition of Complex I (see below).

\section{METFORMIN PREVENTS CELL DEATH WHEN IT IS DUE TO PTP OPENING}

The permeability transition pore (PTP) is a channel located in the inner membrane normally closed in order to maintain a high mitochondrial membrane potential required for ATP synthesis. Once permanently opened, the membrane potential collapses (55), leading to a drastic inhibition of ATP synthesis. Beyond this uncoupling effect, PTP opening has many other effects: It allows the thermodynamic equilibrium of the mitochondrial and cytosolic redox potentials, leading to an increase in cytosolic $\mathrm{NAD}(\mathrm{P}) \mathrm{H}$ concentration (56). It partly inhibits Complex I (57), reallocating the electron flux for the production of reactive oxygen species (58). Finally, it leads to the release of mitochondrial pro-apoptotic proteins both in isolated mitochondria (secondary to mitochondrial swelling leading to the rupture of the outer membrane) (59) and in intact cells (most probably by a distinct but still unknown mechanism) (56, 60-62).

As there are several signaling pathways involved in cell death, there are many factors activating these pathways. To discriminate whether a given condition leading to cell death involves PTP opening or not, experiments are performed in the presence or absence of a recognized PTP inhibitor (generally cyclosporine A, but not exclusively). Using this approach, it has been reproducibly observed that PTP opening occurs when cell death is triggered by calcium overload or oxidative stress (63). 
The molecular nature of the PTP has long been a subject of dispute but recent and compelling data from different laboratories suggest that the PTP might involve ATP synthase $(51,64)$. Surprisingly, the reference Complex I inhibitor rotenone has been shown to inhibit PTP opening in all the tested cells and tissues (either spontaneously or in the presence of cyclosporine A) $(23,65)$. Although rotenone induces an energetic stress, it also prevents cell death in the same models as cyclosporine A (23) and does inhibit Complex I and PTP opening with a similar concentration dependence (65). Piericidin, another well recognized Complex I inhibitor also inhibits PTP opening (23). Thus, the activity of Complex I can be said to be a regulator of PTP opening. Moreover, several ubiquinone analogs (known to bind with Complex I among others) have been proved to regulate PTP opening and cell death $(57,66-69)$.

Knowing that metformin partly inhibits Complex I, we tested whether it also inhibited PTP opening and related cell death. We found that, metformin was less potent than rotenone but also inhibited PTP opening (50). Suggesting a common mechanism of action with rotenone, the effect of metformin was not additive with that of rotenone, whereas it was additive with that of cyclosporine A (65). At present, metformin has been shown to prevent PTP opening in endothelial cells (50), KB cells (7), INS-1 insulinoma cells (61), HeLa cells (65), LNCaP cells (70), A375 cells (70), primary cortical neurons (71) and kidney mitochondria (72). Accordingly, metformin prevents cell death induced by oxidative stress in endothelial cells (50) and KB cells (7), etoposide in primary neurons (71), gentamicin in kidneys (72), hyperglycemia in endothelial (50) and INS-1 cells (61), hyperfructosemia in INS-1 cells (61) and ischemia reperfusion in INS- 1 cells (73). Many other works have found a protective effect of metformin (particularly during oxidative stress or ischemia reperfusion injury) without having studied the role of the PTP $(18,74-77)$.

\section{ANTI-NEOPLASTIC EFFECTS OF METFORMIN}

Although PTP opening irremediably leads to cell death, PTP opening is not mandatory to kill cells as cells can die with a closed PTP. Although Complex I inhibition prevents PTP openingrelated cell death (see above), it can also induce cell death in several models. Indeed, it has been repetitively reported that rotenone (25) or biguanides (including metformin) can induce cell death, especially in cancer cells $(15,17,20,78)$.

Cancer cells are known to be generally highly glycolytic (Warburg effect) and are thus not supposed to be very sensitive to mitochondrial poison. But is it so simple? As soon as cells consume oxygen at the mitochondrial level, they are supposed to produce mitochondrial ATP. Thus, even if the proportion of mitochondrial ATP production is reduced in cancer cells, this mitochondrial ATP production exists and its reduction could be toxic. Supporting this proposal, it has been reported that metformin inhibits the proliferation of HCT116 $\mathrm{p} 53^{-/-}$cancer cells in the presence of glucose, while it induces cell death in case of glucose deprivation (15). Moreover, the effect of metformin is totally prevented by the overexpression of a metformin-resistant Saccharomyces cerevisiae NADH dehydrogenase NDI1 (15), very elegantly demonstrating that the toxicity of metformin is due to its effect on Complex I.

The suggestion that metformin's toxicity is related to an energetic stress raises several questions: Why is metformin less toxic in non-cancer cells that are yet more dependent on mitochondrial ATP production? How can metformin protect against PTP-induced cell death despite its effect on ATP production? In other words, what triggers that a same inhibition of Complex I either prevents or induces cell death?

Again, part of the answer could be found in the comparison of metformin concentrations. While millimolar concentrations of metformin are generally used to induce cell death in vitro, micromolar concentrations are sufficient to prevent PTPopening induced cell death. Although it has been shown that cellular energy status is inversely correlated with metformin concentrations (11, 79), a 24-h incubation with $100 \mu \mathrm{M}$ metformin did not affect the AMP/ATP ratio in primary cultured hepatocytes (11). This suggests that the metformin concentration used to prevent PTP opening $(100 \mu \mathrm{M}$, overnight $)$ was not sufficient to induce a lethal decrease in energy status. On the contrary, this confirms that the concentrations used to kill cells dramatically affect the energy status. Note however that some authors have reported an anti-apoptotic effect even at millimolar concentrations of metformin, suggesting that some cells are able to overcome energy stress $(75,80)$.

However, if the mechanism by which metformin kills isolated cells can be traced to a collapse in energy status, the concentrations that prevent cancer growth in animal models are in the micromolar range. The practical assumption of metformin accumulation in mitochondria has obviously been retained, but one can wonder: why are normal cells preserved? Alternative or complementary explanations must exist. Among them, it has been proposed that the effect of metformin in animal models is indirect (for example due to a decrease in blood insulin concentration) (2). It is also possible that the accumulation of metformin or the sensitivity of Complex I to metformin is higher in cancer cells than in normal tissues (personal hypothesis). As far as I know, these assumptions have not yet been tested.

\section{CONCLUSIONS AND PROPOSAL}

As explained several times in this manuscript, the concentration with which experiments were conducted is the main misleading point regarding the effect of metformin on Complex I. On the one hand, it is obvious that the assumption that metformin accumulates in mitochondria suits many authors. This hypothesis can bridge the gap between concentrations measured in vivo and those used in vitro. On the other hand, two different laboratories that attempted to measure such an accumulation put forward a total absence of metformin accumulation in mitochondria $(6,45)$ in which Complex I was nevertheless inhibited (6). Furthermore, although the pharmacokinetic data are indirect evidence, they are not compatible with an accumulation of metformin in mitochondria. 
Facing the facts, one must admit that there is either a technical mistake in the studies that did not find metformin accumulation in mitochondria or there is absolutely no experiment performed at millimolar concentrations of metformin that reflect what occurs in vivo. This includes a lot of articles both on its antidiabetic role and on its anticancer effect. There is an urgent need to solve this problem for good, and this could be performed easily by fast cell fractionation coupled to mass spectrometry (or other technics to detect metformin) in order to confirm if metformin is found in large amount in mitochondria of cells exposed to metformin. Currently, the published evidence does not support the

\section{REFERENCES}

1. Li M, Li X, Zhang H, Lu Y. Molecular mechanisms of metformin for diabetes and cancer treatment. Front Physiol. (2018) 9:1039. doi: 10.3389/fphys.2018.01039

2. Yu X, Mao W, Zhai Y, Tong C, Liu M, Ma L, et al. Anti-tumor activity of metformin: from metabolic and epigenetic perspectives. Oncotarget (2017) 8:5619-28. doi: 10.18632/oncotarget.13639

3. Rotermund C, Machetanz G, Fitzgerald JC. The therapeutic potential of metformin in neurodegenerative diseases. Front Endocrinol (Lausanne). (2018) 9:400. doi: 10.3389/fendo.2018.00400

4. El-Mir MY, Nogueira V, Fontaine E, Averet N, Rigoulet M, Leverve X. Dimethylbiguanide inhibits cell respiration via an indirect effect targeted on the respiratory chain complex I. J Biol Chem. (2000) 275:223-8. doi: $10.1074 /$ jbc.275.1.223

5. Owen MR, Doran E, Halestrap AP. Evidence that metformin exerts its anti-diabetic effects through inhibition of complex 1 of the mitochondrial respiratory chain. Biochem J. (2000) 348:607-14. doi: 10.1042/bj3480607

6. Detaille D, Guigas B, Leverve X, Wiernsperger N, Devos P. Obligatory role of membrane events in the regulatory effect of metformin on the respiratory chain function. Biochem Pharmacol. (2002) 63:1259-72. doi: 10.1016/S0006-2952(02)00858-4

7. Guigas B, Detaille D, Chauvin C, Batandier C, De Oliveira F, Fontaine E, et al. Metformin inhibits mitochondrial permeability transition and cell death: a pharmacological in vitro study. Biochem J. (2004) 382:877-84. doi: 10.1042/BJ20040885

8. Carvalho C, Correia S, Santos MS, Seica R, Oliveira CR, Moreira PI. Metformin promotes isolated rat liver mitochondria impairment. Mol Cell Biochem. (2008) 308:75-83. doi: 10.1007/s11010-007-9614-3

9. Dykens JA, Jamieson J, Marroquin L, Nadanaciva S, Billis PA, Will Y. Biguanide-induced mitochondrial dysfunction yields increased lactate production and cytotoxicity of aerobically-poised HepG2 cells and human hepatocytes in vitro. Toxicol Appl Pharmacol. (2008) 233:203-10. doi: 10.1016/j.taap.2008.08.013

10. Palenickova E, Cahova M, Drahota Z, Kazdova L, Kalous M. Inhibitory effect of metformin on oxidation of NADH-dependent substrates in rat liver homogenate. Physiol Res. (2011) 60:835-9.

11. Stephenne X, Foretz M, Taleux N, van der Zon GC, Sokal E, Hue L, et al. Metformin activates AMP-activated protein kinase in primary human hepatocytes by decreasing cellular energy status. Diabetologia (2011) 54:310110. doi: 10.1007/s00125-011-2311-5

12. Hirsch A, Hahn D, Kempna P, Hofer G, Nuoffer JM, Mullis PE, et al. Metformin inhibits human androgen production by regulating steroidogenic enzymes HSD3B2 and CYP17A1 and complex I activity of the respiratory chain. Endocrinology (2012) 153:4354-66. doi: 10.1210/en.2012-1145

13. Bridges HR, Jones AJ, Pollak MN, Hirst J. Effects of metformin and other biguanides on oxidative phosphorylation in mitochondria. Biochem J. (2014) 462:475-87. doi: 10.1042/BJ20140620

14. Wessels B, Ciapaite J, van den Broek NM, Nicolay K, Prompers JJ. Metformin impairs mitochondrial function in skeletal muscle of both lean generally accepted hypothesis of metformin accumulation in mitochondria.

\section{AUTHOR CONTRIBUTIONS}

The author confirms being the sole contributor of this work and has approved it for publication.

\section{ACKNOWLEDGMENTS}

I thank Christophe Cottet and Emily Tubbs for the corrections of the English version of this paper. and diabetic rats in a dose-dependent manner. PLoS ONE (2014) 9:e100525. doi: 10.1371/journal.pone.0100525

15. Wheaton WW, Weinberg SE, Hamanaka RB, Soberanes S, Sullivan LB, Anso E, et al. Metformin inhibits mitochondrial complex I of cancer cells to reduce tumorigenesis. Elife (Cambridge) (2014) 3:e02242. doi: 10.7554/eLife.02242

16. Matsuzaki S, Humphries KM. Selective inhibition of deactivated mitochondrial complex I by biguanides. Biochemistry (2015) 54:2011-21. doi: 10.1021/bi501473h

17. Boukalova S, Stursa J, Werner L, Ezrova Z, Cerny J, BezaworkGeleta A, et al. Mitochondrial targeting of metformin enhances its activity against pancreatic cancer. Mol Cancer Ther. (2016) 15:2875-86. doi: 10.1158/1535-7163.MCT-15-1021

18. Ling S, Shan Q, Liu P, Feng T, Zhang X, Xiang P, et al. Metformin ameliorates arsenic trioxide hepatotoxicity via inhibiting mitochondrial complex I. Cell Death Dis. (2017) 8:e3159. doi: 10.1038/cddis.2017.482

19. Cameron AR, Logie L, Patel K, Erhardt S, Bacon S, Middleton P, et al. Metformin selectively targets redox control of complex I energy transduction. Redox Biol. (2018) 14:187-97. doi: 10.1016/j.redox.2017.08.018

20. Wu Y, Gao WN, Xue YN, Zhang LC, Zhang JJ, Lu SY, et al. SIRT3 aggravates metformin-induced energy stress and apoptosis in ovarian cancer cells. Exp. Cell Res. (2018) 367:137-49 doi: 10.1016/j.yexcr.2018.03.030

21. Pryor HJ, Smyth JE, Quinlan PT, Halestrap AP. Evidence that the flux control coefficient of the respiratory chain is high during gluconeogenesis from lactate in hepatocytes from starved rats. Implications for the hormonal control of gluconeogenesis and action of hypoglycaemic agents. Biochem J. (1987) 247:449-57. doi: 10.1042/bj2470449

22. Hou WL, Yin J, Alimujiang $M$, Yu XY, Ai LG, Bao YQ, et al. Inhibition of mitochondrial complex I improves glucose metabolism independently of AMPK activation. J Cell Mol Med. (2018) 22:1316-28. doi: $10.1111 /$ jcmm.13432

23. Chauvin C, De Oliveira F, Ronot X, Mousseau M, Leverve X, Fontaine E. Rotenone inhibits the mitochondrial permeability transition-induced cell death in U937 and KB cells. J Biol Chem. (2001) 276:41394-8. doi: 10.1074/jbc.M106417200

24. Li N, Ragheb K, Lawler G, Sturgis J, Rajwa B, Melendez JA, et al. Mitochondrial complex I inhibitor rotenone induces apoptosis through enhancing mitochondrial reactive oxygen species production. J Biol Chem. (2003) 278:8516-25. doi: 10.1074/jbc.M210432200

25. Deng YT, Huang HC, Lin JK. Rotenone induces apoptosis in MCF-7 human breast cancer cell-mediated ROS through JNK and p38 signaling. Mol Carcinog. (2010) 49:141-51. doi: 10.1002/mc.20583

26. Xu A, Szczepanek K, Maceyka MW, Ross T, Bowler E, Hu Y, et al. Transient complex I inhibition at the onset of reperfusion by extracellular acidification decreases cardiac injury. Am J Physiol Cell Physiol. (2014) 306:C1142-53. doi: 10.1152/ajpcell.00241.2013

27. Hu W, Tian H, Yue W, Li L, Li S, Gao C, et al. Rotenone induces apoptosis in human lung cancer cells by regulating autophagic flux. IUBMB Life (2016) 68:388-93. doi: 10.1002/iub.1493

28. Rekuviene E, Ivanoviene L, Borutaite V, Morkuniene R. Rotenone decreases ischemia-induced injury by inhibiting mitochondrial 
permeability transition in mature brains. Neurosci Lett. (2017) 653:45-50. doi: 10.1016/j.neulet.2017.05.028

29. Gong L, Goswami S, Giacomini KM, Altman RB, Klein TE. Metformin pathways: pharmacokinetics and pharmacodynamics. Pharmacogenet Genomics (2012) 22:820-7. doi: 10.1097/FPC.0b013e3283559b22

30. Shu Y, Sheardown SA, Brown C, Owen RP, Zhang S, Castro RA, et al. Effect of genetic variation in the organic cation transporter 1 (OCT1) on metformin action. J Clin Invest. (2007) 117:1422-31. doi: 10.1172/JCI 30558

31. Higgins JW, Bedwell DW, Zamek-Gliszczynski MJ. Ablation of both organic cation transporter (OCT) 1 and OCT2 alters metformin pharmacokinetics but has no effect on tissue drug exposure and pharmacodynamics. Drug Metab Dispos. (2012) 40:1170-7. doi: 10.1124/dmd.112.044875

32. Jensen JB, Sundelin EI, Jakobsen S, Gormsen LC, Munk OL, Frokiaer J, et al. [11C]-Labeled metformin distribution in the liver and small intestine using dynamic positron emission tomography in mice demonstrates tissue-specific transporter dependency. Diabetes (2016) 65:1724-30. doi: 10.2337/db16-0032

33. Wang DS, Jonker JW, Kato Y, Kusuhara H, Schinkel AH, Sugiyama Y. Involvement of organic cation transporter 1 in hepatic and intestinal distribution of metformin. J Pharmacol Exp Ther. (2002) 302:510-5. doi: $10.1124 /$ jpet.102.034140

34. Tsuda M, Terada T, Mizuno T, Katsura T, Shimakura J, Inui K. Targeted disruption of the multidrug and toxin extrusion 1 (matel) gene in mice reduces renal secretion of metformin. Mol Pharmacol. (2009) 75:1280-6. doi: $10.1124 / \mathrm{mol} .109 .056242$

35. Shu Y, Brown C, Castro RA, Shi RJ, Lin ET, Owen RP, et al. Effect of genetic variation in the organic cation transporter 1, OCT1, on metformin pharmacokinetics. Clin Pharmacol Ther. (2008) 83:273-80. doi: 10.1038/sj.clpt.6100275

36. Christensen MM, Brasch-Andersen C, Green H, Nielsen F, Damkier $\mathrm{P}$, Beck-Nielsen $\mathrm{H}$, et al. The pharmacogenetics of metformin and its impact on plasma metformin steady-state levels and glycosylated hemoglobin A1c. Pharmacogenet Genomics (2011) 21:837-50. doi: 10.1097/FPC.0b013e32834c0010

37. Shikata E, Yamamoto R, Takane H, Shigemasa C, Ikeda T, Otsubo K, et al. Human organic cation transporter (OCT1 and OCT2) gene polymorphisms and therapeutic effects of metformin. J Hum Genet. (2007) 52:117-22. doi: 10.1007/s10038-006-0087-0

38. Zhou K, Donnelly LA, Kimber CH, Donnan PT, Doney AS, Leese G, et al. Reduced-function SLC22A1 polymorphisms encoding organic cation transporter 1 and glycemic response to metformin: a GoDARTS study. Diabetes (2009) 58:1434-9. doi: 10.2337/db08-0896

39. Funk RS, Krise JP. Cationic amphiphilic drugs cause a marked expansion of apparent lysosomal volume: implications for an intracellular distribution-based drug interaction. Mol Pharm. (2012) 9:1384-95. doi: $10.1021 / \mathrm{mp} 200641 \mathrm{e}$

40. Pentikainen PJ, Neuvonen PJ, Penttila A. Pharmacokinetics of metformin after intravenous and oral administration to man. Eur J Clin Pharmacol. (1979) 16:195-202. doi: 10.1007/BF00562061

41. Tucker GT, Casey C, Phillips PJ, Connor H, Ward JD, Woods HF. Metformin kinetics in healthy subjects and in patients with diabetes mellitus. $\mathrm{Br}$ J Clin Pharmacol. (1981) 12:235-46. doi: 10.1111/j.1365-2125.1981.tb01206.x

42. Scheen AJ. Clinical pharmacokinetics of metformin. Clin Pharmacokinet. (1996) 30:359-71. doi: 10.2165/00003088-199630050-00003

43. Gormsen LC, Sundelin EI, Jensen JB, Vendelbo MH, Jakobsen S, Munk $\mathrm{OL}$, et al. In vivo imaging of human 11c-metformin in peripheral organs: dosimetry, biodistribution, and kinetic analyses. J Nucl Med. (2016) 57:19206. doi: 10.2967/jnumed.116.177774

44. Rena G, Hardie DG, Pearson ER. The mechanisms of action of metformin. Diabetologia (2017) 60:1577-85. doi: 10.1007/s00125-017-4342-z

45. Wilcock C, Wyre ND, Bailey CJ. Subcellular distribution of metformin in rat liver. J Pharm Pharmacol. (1991) 43:442-4. doi: 10.1111/j.2042-7158.1991.tb03507.x

46. Davidoff $F$. Effects of guanidine derivatives on mitochondrial function. 3. The mechanism of phenethylbiguanide accumulation and its relationship to in vitro respiratory inhibition. J Biol Chem. (1971) 246:4017-27.

47. Cheng G, Zielonka J, Ouari O, Lopez M, McAllister D, Boyle K, et al. Mitochondria-targeted analogues of metformin exhibit enhanced antiproliferative and radiosensitizing effects in pancreatic cancer cells. Cancer Res. (2016) 76:3904-15. doi: 10.1158/0008-5472.CAN-15-2534

48. Boyle KA, Van Wickle J, Hill RB, Marchese A, Kalyanaraman B, Dwinell MB. Mitochondria-targeted drugs stimulate mitophagy and abrogate colon cancer cell proliferation. J Biol Chem. (2018) 293:14891-904. doi: 10.1074/jbc.RA117.001469

49. Schafer G. Site-specific uncoupling and inhibition of oxidative phosphorylation by biguanides. II. Biochim Biophys Acta (1969) 172:334-7. doi: 10.1016/0005-2728(69)90077-2

50. Detaille D, Guigas B, Chauvin C, Batandier C, Fontaine E, Wiernsperger N, et al. Metformin prevents high-glucose-induced endothelial cell death through a mitochondrial permeability transition-dependent process. Diabetes (2005) 54:2179-87. doi: 10.2337/diabetes.54.7.2179

51. Giorgio V, von Stockum S, Antoniel M, Fabbro A, Fogolari F, Forte M, et al. Dimers of mitochondrial ATP synthase form the permeability transition pore. Proc Natl Acad Sci USA. (2013) 110:5887-92. doi: 10.1073/pnas.1217823110

52. Vinogradov AD, Grivennikova VG. The mitochondrial complex I: progress in understanding of catalytic properties. IUBMB Life (2001) 52:129-34. doi: $10.1080 / 15216540152845920$

53. Galkin A, Meyer B, Wittig I, Karas M, Schagger H, Vinogradov A, et al. Identification of the mitochondrial ND3 subunit as a structural component involved in the active/deactive enzyme transition of respiratory complex I. $J$ Biol Chem. (2008) 283:20907-13. doi: 10.1074/jbc.M803190200

54. Hurd TR, Requejo R, Filipovska A, Brown S, Prime TA, Robinson AJ, et al. Complex I within oxidatively stressed bovine heart mitochondria is glutathionylated on Cys-531 and Cys-704 of the $75-\mathrm{kDa}$ subunit: potential role of CYS residues in decreasing oxidative damage. J Biol Chem. (2008) 283:24801-15. doi: 10.1074/jbc.M803432200

55. Petronilli V, Penzo D, Scorrano L, Bernardi P, Di Lisa F. The mitochondrial permeability transition, release of cytochrome $c$ and cell death. Correlation with the duration of pore openings in situ. J Biol Chem. (2001) 276:12030-4. doi: $10.1074 /$ jbc.M010604200

56. Dumas JF, Argaud L, Cottet-Rousselle C, Vial G, Gonzalez C, Detaille D, et al. Effect of transient and permanent permeability transition pore opening on NAD(P)H localization in intact cells. J Biol Chem. (2009) 284:15117-25. doi: 10.1074/jbc.M900926200

57. Fontaine E, Eriksson O, Ichas F, Bernardi P. Regulation of the permeability transition pore in skeletal muscle mitochondria. Modulation by electron flow through the respiratory chain complex i. J Biol Chem. (1998) 273:12662-8. doi: $10.1074 /$ jbc.273.20.12662

58. Batandier C, Leverve X, Fontaine E. Opening of the mitochondrial permeability transition pore induces reactive oxygen species production at the level of the respiratory chain complex I. J Biol Chem. (2004) 279:17197-204. doi: $10.1074 /$ jbc.M310329200

59. Kantrow SP, Piantadosi CA. Release of cytochrome $\mathrm{c}$ from liver mitochondria during permeability transition. Biochem Biophys Res Commun. (1997) 232:669-71. doi: 10.1006/bbrc.1997.6353

60. De Giorgi F, Lartigue L, Bauer MK, Schubert A, Grimm S, Hanson GT, et al. The permeability transition pore signals apoptosis by directing Bax translocation and multimerization. FASEB J. (2002) 16:607-9. doi: 10.1096/fj.01-0269fje

61. Lablanche S, Cottet-Rousselle C, Lamarche F, Benhamou PY, Halimi S, Leverve X, et al. Protection of pancreatic INS-1 beta-cells from glucoseand fructose-induced cell death by inhibiting mitochondrial permeability transition with cyclosporin A or metformin. Cell Death Dis. (2011) 2:e134. doi: $10.1038 /$ cddis. 2011.15

62. Lamarche F, Carcenac C, Gonthier B, Cottet-Rousselle C, Chauvin C, Barret L, et al. Mitochondrial permeability transition pore inhibitors prevent ethanol-induced neuronal death in mice. Chem Res Toxicol. (2013) 26:78-88. doi: $10.1021 /$ tx $300395 \mathrm{w}$

63. Rasola A, Bernardi P. Mitochondrial permeability transition in $\mathrm{Ca}(2+)$ dependent apoptosis and necrosis. Cell Calcium (2011) 50:222-33. doi: 10.1016/j.ceca.2011.04.007

64. Alavian KN, Beutner G, Lazrove E, Sacchetti S, Park HA, Licznerski $\mathrm{P}$, et al. An uncoupling channel within the c-subunit ring of the F1FO ATP synthase is the mitochondrial permeability transition pore. Proc Natl Acad Sci USA. (2014) 111:10580-5. doi: 10.1073/pnas.14015 91111 
65. Li B, Chauvin C, De Paulis D, De Oliveira F, Gharib A, Vial G, et al. Inhibition of complex I regulates the mitochondrial permeability transition through a phosphate-sensitive inhibitory site masked by cyclophilin D. Biochim Biophys Acta (2012) 1817:1628-34. doi: 10.1016/j.bbabio.2012.05.011

66. Walter L, Nogueira V, Leverve X, Heitz MP, Bernardi P, Fontaine E. Three classes of ubiquinone analogs regulate the mitochondrial permeability transition pore through a common site. J Biol Chem. (2000) 275:29521-7. doi: $10.1074 /$ jbc.M004128200

67. Walter L, Miyoshi H, Leverve X, Bernard P, Fontaine E. Regulation of the mitochondrial permeability transition pore by ubiquinone analogs. A progress report. Free Radic Res. (2002) 36:405-12. doi: 10.1080/10715760290021252

68. Devun F, Walter L, Belliere J, Cottet-Rousselle C, Leverve X, Fontaine E. Ubiquinone analogs: a mitochondrial permeability transition poredependent pathway to selective cell death. PLOS ONE (2010) 5:e11792. doi: 10.1371/journal.pone.0011792

69. Belliere J, Devun F, Cottet-Rousselle C, Batandier C, Leverve X, Fontaine E. Prerequisites for ubiquinone analogs to prevent mitochondrial permeability transition-induced cell death. J Bioenerg Biomembr. (2012) 44:207-12. doi: 10.1007/s10863-012-9406-7

70. Loubiere C, Clavel S, Gilleron J, Harisseh R, Fauconnier J, Ben-Sahra I, et al. The energy disruptor metformin targets mitochondrial integrity via modification of calcium flux in cancer cells. Sci Rep. (2017) 7:5040. doi: 10.1038/s41598-017-05052-2

71. El-Mir MY, Detaille D, R-Villanueva G, Delgado-Esteban M, Guigas B, Attia $\mathrm{S}$, et al. Neuroprotective role of antidiabetic drug metformin against apoptotic cell death in primary cortical neurons. J Mol Neurosci. (2008) 34:77-87. doi: 10.1007/s12031-007-9002-1

72. Morales AI, Detaille D, Prieto M, Puente A, Briones E, Arevalo M, et al. Metformin prevents experimental gentamicin-induced nephropathy by a mitochondria-dependent pathway. Kidney Int. (2010) 77:861-9. doi: 10.1038/ki.2010.11

73. Lablanche S, Cottet-Rousselle C, Argaud L, Laporte C, Lamarche F, Richard MJ, et al. Respective effects of oxygen and energy substrate deprivation on beta cell viability. Biochim Biophys Acta (2015) 1847:629-39. doi: 10.1016/j.bbabio.2015.04.002
74. Elmadhun NY, Sabe AA, Lassaletta AD, Chu LM, Sellke FW. Metformin mitigates apoptosis in ischemic myocardium. J Surg Res. (2014) 192:50-8. doi: 10.1016/j.jss.2014.05.005

75. Cahova M, Palenickova E, Dankova H, Sticova E, Burian M, Drahota Z, et al. Metformin prevents ischemia reperfusion-induced oxidative stress in the fatty liver by attenuation of reactive oxygen species formation. Am J Physiol Gastrointest Liver Physiol. (2015) 309:G100-11. doi: 10.1152/ajpgi.00329.2014

76. Chen D, Xia D, Pan Z, Xu D, Zhou Y, Wu Y, et al. Metformin protects against apoptosis and senescence in nucleus pulposus cells and ameliorates disc degeneration in vivo. Cell Death Dis. (2016) 7:e2441. doi: $10.1038 /$ cddis.2016.334

77. Wang X, Yang L, Kang L, Li J, Yang L, Zhang J, et al. Metformin attenuates myocardial ischemia-reperfusion injury via up-regulation of antioxidant enzymes. PLoS ONE (2017) 12:e0182777. doi: 10.1371/journal.pone.0182777

78. Gong J, Robbins LA, Lugea A, Waldron RT, Jeon CY, Pandol SJ. Diabetes, pancreatic cancer, and metformin therapy. Front Physiol. (2014) 5:426. doi: 10.3389/fphys.2014.00426

79. Foretz M, Hebrard S, Leclerc J, Zarrinpashneh E, Soty M, Mithieux G, et al. Metformin inhibits hepatic gluconeogenesis in mice independently of the LKB1/AMPK pathway via a decrease in hepatic energy state. J Clin Invest. (2010) 120:2355-69. doi: 10.1172/JCI40671

80. Bibi F, Ullah I, Kim MO, Naseer MI. Metformin attenuate PTZ-induced apoptotic neurodegeneration in human cortical neuronal cells. Pak J Med Sci. (2017) 33:581-5. doi: 10.12669/pjms.333.11996

Conflict of Interest Statement: The author declares that the research was conducted in the absence of any commercial or financial relationships that could be construed as a potential conflict of interest.

Copyright (๑) 2018 Fontaine. This is an open-access article distributed under the terms of the Creative Commons Attribution License (CC BY). The use, distribution or reproduction in other forums is permitted, provided the original author(s) and the copyright owner(s) are credited and that the original publication in this journal is cited, in accordance with accepted academic practice. No use, distribution or reproduction is permitted which does not comply with these terms. 